\title{
Proposta de Suporte à Parametrização no NPB com CUDA
}

\author{
Gabriell Araujo, Dalvan Griebler, Luiz G. Fernandes \\ ${ }^{1}$ Escola Politécnica, Grupo de Modelagem de Aplicações Paralelas (GMAP), \\ Pontifícia Universidade Católica do Rio Grande do Sul (PUCRS), Porto Alegre, Brasil \\ gabriell.araujodedu.pucrs.br, \{dalvan.griebler,luiz.fernandes\}@pucrs.br
}

Resumo. Este trabalho propõe a introdução de parâmetros configuráveis para GPUs no NPB. A etapa inicial do estudo contemplou a parametrização do número de threads por bloco e seu impacto no desempenho de GPUs.

\section{Contexto}

As unidades de processamento gráfico (GPUs) são arquiteturas populares e oferecem paralelismo massivo a baixo custo, porém, sua utilização eficiente é um desafio. Benchmarks são uma forma de suportar a pesquisa de GPUs. NAS Parallel Benchmarks (NPB) é um consolidado conjunto de benchmarks baseado em computações de dinâmica de fluidos [Bailey et al. 1994] (CFD) que está recebendo atenção no contexto de GPUs com artigos publicados recentemente [Do et al. 2019, d. Araujo et al. 2020].

Uma maneira eficiente de fornecer melhores informações sobre o desempenho em benchmarks é oferecer parâmetros configuráveis. Nesse sentido, este trabalho propõe a introdução de parâmetros para GPUs no NPB, iniciando pela parametrização do número de threads por bloco e avaliação do seu impacto no desempenho.

\section{NPB com CUDA e Número Configurável de Threads}

A implementação seguiu boas práticas de programação para GPUs. Ao usuário é permitido especificar o número de threads por bloco, através de um arquivo de texto com os valores para cada uma das funções que são paralelizadas para GPU. Os experimentos foram realizados em uma máquina equipada com uma GPU NVIDIA Titan X Pascal (3584 CUDA Cores). A classe $C$ foi utilizada como carga de trabalho. Cada teste foi repetido dez vezes. Foram exploradas cinco estratégias para a escolha do número de threads. Warp utiliza o tamanho do warp da GPU. Max utiliza o número máximo de threads por bloco suportado pela GPU. Manual utiliza a "intuição" do programador. Profiling realiza profiling individual de cada uma das funções da GPU, e coleta o melhor valor para cada função. Exhausting testa todas as combinações possíveis de threads e coleta a combinação que apresenta o menor tempo de execução.

As estratégias foram aplicadas na implementação deste trabalho e também foram realizados experimentos com os trabalhos da literatura (valores estáticos, pois não suportam parametrização). Cada versão do NPB é nomeada como segue: Versão sequencial [Griebler et al. 2018] como Serial. Versão OpenMP [Griebler et al. 2018] como OpenMP. Versão OpenCL [Seo et al. 2011] como OpenCL. Versão OpenCL [Do et al. 2019] como OpenCL-2019. Versão OpenACC [Xu et al. 2015] como OpenACC. Versão CUDA [d. Araujo et al. 2020] como CUDA. Versão CUDA [Do et al. 2019] como CUDA-2019. Versão CUDA deste trabalho com cinco diferentes estratégias como Warp, Max, Manual, Profiling e Exhausting. 
A Figura 1 apresenta o tempo de execução e o desvio padrão através de barras de erro. Foi observado aperfeiçoamento de desempenho relevante em três benchmarks ao mudar as estratégias de threads, até $17 \%$ em EP, $105 \%$ em BT, e $554 \%$ em CG. EP consome um grande número de registradores e obtém melhor desempenho com quantidades menores de threads. BT possui overhead de sincronizações que é aliviado ao utilizar quantidades maiores de threads. CG gera pequenas tarefas de tamanhos aleató-

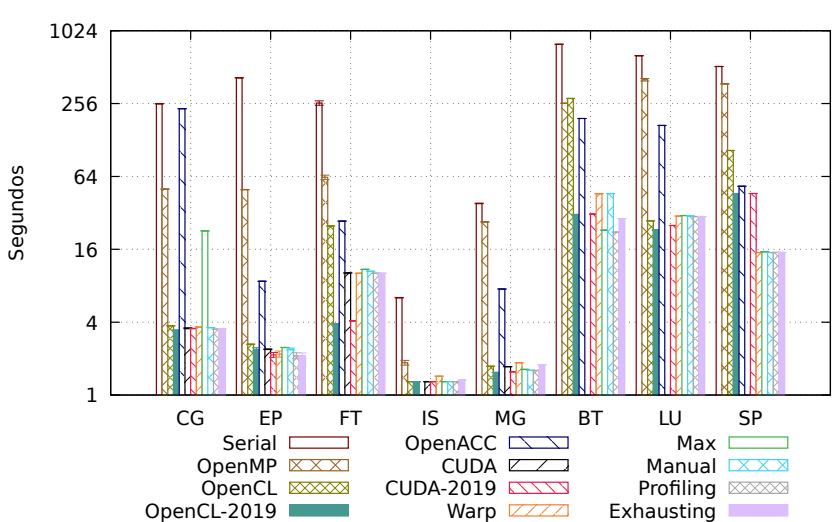

Figura 1. Tempo de execução com o desvio padrão. rios, e ao utilizar-se grandes quantidades de threads, muitas delas permanecem inativas. Os demais benchmarks sofreram impactos menores, pois apresentam limitações moderadas.

\section{Conclusões}

Demonstrou-se que o número de threads pode impactar no desempenho dos benchmarks e também fornecer informações relevantes sobre o software e o hardware. Como trabalhos futuros, novos parâmetros para GPUs podem ser adicionados e avaliados no NPB.

\section{Referências}

Bailey, D. H., Barszcz, E., Barton, J. T., Browning, D. S., Carter, R. L., Fatoohi, R. A., Frederickson, P. O., Lasinski, T. A., Simon, H. D., Venkatakrishnan, V., and Weeratunga, S. K. (1994). The NAS Parallel Benchmarks RNR-94-007. Technical report, NASA Advanced Supercomputing Division.

d. Araujo, G. A., Griebler, D., Danelutto, M., and Fernandes, L. G. (2020). Efficient NAS Parallel Benchmark Kernels with CUDA. In 2020 28th Euromicro International Conference on Parallel, Distributed and Network-Based Processing (PDP), pages 916.

Do, Y., Kim, H., Oh, P., Park, D., and Lee, J. (2019). SNU-NPB 2019: Parallelizing and Optimizing NPB in OpenCL and CUDA for Modern GPUs. In 2019 IEEE International Symposium on Workload Characterization (IISWC), pages 93-105.

Griebler, D., Loff, J., Mencagli, G., Danelutto, M., and Fernandes, L. G. (2018). Efficient NAS Benchmark Kernels with C++ Parallel Programming. In 26th Euromicro International Conference on Parallel, Distributed and Network-Based Processing (PDP), PDP'18, pages 733-740, Cambridge, UK. IEEE.

Seo, S., Jo, G., and Lee, J. (2011). Performance Characterization of the NAS Parallel Benchmarks in OpenCL. In 2011 IEEE International Symposium on Workload Characterization (IISWC), pages $137-148$.

Xu, R., Tian, X., Chandrasekaran, S., Yan, Y., and Chapman, B. (2015). NAS Parallel Benchmarks for GPGPUs Using a Directive-Based Programming Model. In Brodman, J. and Tu, P., editors, Languages and Compilers for Parallel Computing, pages 67-81, Cham. Springer International Publishing. 